\title{
Spatial dependence of plasma oscillations in Josephson tunnel junctions
}

\author{
Holst, Thorsten; Hansen, Jørn Bindslev
}

Published in:

Physical Review B

Link to article, DOI:

10.1103/PhysRevB.44.2238

Publication date:

1991

Document Version

Publisher's PDF, also known as Version of record

Link back to DTU Orbit

Citation (APA):

Holst, T., \& Hansen, J. B. (1991). Spatial dependence of plasma oscillations in Josephson tunnel junctions. Physical Review B, 44(5), 2238-2245. https://doi.org/10.1103/PhysRevB.44.2238

\section{General rights}

Copyright and moral rights for the publications made accessible in the public portal are retained by the authors and/or other copyright owners and it is a condition of accessing publications that users recognise and abide by the legal requirements associated with these rights.

- Users may download and print one copy of any publication from the public portal for the purpose of private study or research.

- You may not further distribute the material or use it for any profit-making activity or commercial gain

- You may freely distribute the URL identifying the publication in the public portal

If you believe that this document breaches copyright please contact us providing details, and we will remove access to the work immediately and investigate your claim. 


\title{
Spatial dependence of plasma oscillations in Josephson tunnel junctions
}

\author{
Thorsten Holst and J. Bindslev Hansen \\ Physics Laboratory I/MIDIT, The Technical University of Denmark, DK-2800 Lyngby, Denmark
}

(Received 5 December 1990)

\begin{abstract}
We report on direct measurements of the plasma oscillations in Josephson tunnel junctions of various spatial dimensions. The effect of the spatial variation of the Cooper-pair phase difference (the Josephson phase) on the dynamics of the junction was investigated by application of a static magnetic field threading the tunneling barrier. We compare measurements where the plasma frequency was tuned either by applying a magnetic field or by raising the temperature. A crossover from short- to long-junction behavior of the functional dependence of the plasma oscillations was observed in the case of an applied magnetic field. Numerical simulations of the governing partial-differential sine-Gordon equation were performed and compared to the experimental results and a perturbation analysis. The theoretical results support the experiments and allow us to interpret the observed crossover as due to the spatial variation of the Josephson phase.
\end{abstract}

\section{INTRODUCTION}

The main purpose of this work is to study the influence of the spatial dimension on the dynamics of Josephson tunnel junctions. In order to investigate this spatial dependence of the Josephson phase, we have applied a magnetic field in the plane of the tunneling barrier and carried out experiments on junctions of various normalized lengths. A Josephson junction is called short if both the length $L$ and the width $W$ of the tunneling area are short compared to the Josephson penetration depth, $\lambda_{J}$. We have long, quasi-one-dimensional junctions if one of the physical dimensions is larger than $\lambda_{J}$, i.e., $W<<\lambda_{J}<L$.

The plasma oscillations in the zero-voltage state were chosen for the study as a measure of the basic dynamics of the junction. Plasma oscillations in Josephson tunnel junctions, first reported by Dahm et al., ${ }^{1}$ involve a resonant exchange of energy between the electric field and the kinetic energy of the Cooper pairs. With typical junction parameters the plasma frequency may range from 1 to $100 \mathrm{GHz}$. The plasma frequency plays an important role in the study of nonlinear dynamics in Josephson junctions, where the ratio between the frequency of the driving signal and the plasma frequency is an important parameter. ${ }^{2}$ Some noise properties, such as the lifetime of the zero-voltage state, are also affected by the plasma frequency of the junction. ${ }^{3}$ To keep things simple we will only be concerned with small amplitude plasma oscillations. Measurements of large amplitude plasma oscillations in the nonlinear regime are reported in Refs. 1, 4, and 5 .

In the next section we present the model along with the theory of plasma oscillations, both for short and long junctions. The experimental procedure and results are reported in Secs. III and IV, followed in Sec. V by numerical simulations and comparison with a perturbation result. Finally we discuss our results.

\section{THEORY}

The theoretical concept of plasma oscillations is based on the simple and widely used resistor-capacitor shunted junction (RCSJ) model. The current of Cooper pairs is modeled by the Josephson equations, while the loss, due to thermally excited pairs, is represented by a shunt conductance $1 / R$. A shunt capacitance $C$ is formed by the geometric overlap of the two superconducting electrodes (see Fig. 1). Since the impedance of the junction is much smaller than the impedance of the external circuit both in the microwave regime and in the dc power circuit, we may assume that current sources, $I(t)$, are used to bias

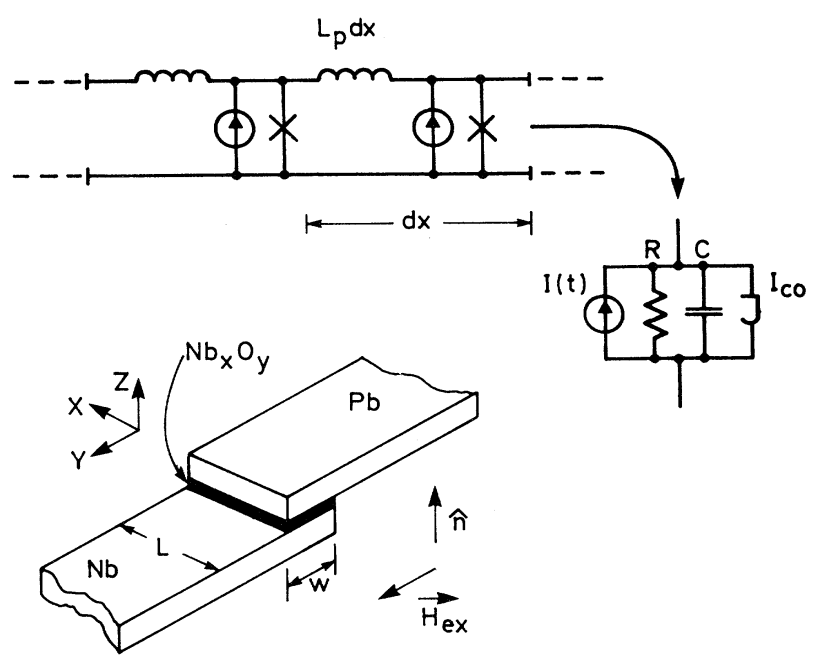

FIG. 1. A schematic drawing of the Josephson tunnel junction is shown in the bottom part of the figure. The equivalent diagram for a short RCSJ junction, $L, W<<\lambda_{J}$, is shown to the right. The transmission-line equivalent for a long onedimensional junction, $W<<\lambda_{J}<L$, consists of infinitesimally small RCSJ circuits connected by the inductance $L_{p} d x$. 
the junction.

In Sec. II A we will shortly review the theory of plasma oscillations in short junctions. A simple generalization is made to cover the case where we have a symmetric variation of the critical density over the junction. In the next section we consider longer junctions where the spatial structure of the junction must be taken into account. A perturbation result is presented for this case.

\section{A. Short junctions}

The equation of motion for the Josephson phase, $\phi$, is obtained by calculating the various current contributions through the tunneling barrier and using the Josephson equations 6

$$
I_{J}(t)=I_{c 0} \sin \phi(t), \quad V(t)=\frac{\hbar}{2 e} \phi_{t},
$$

where $I_{J}$ is the Cooper-pair current, $I_{c 0}$ is the critical current, and $V$ is the voltage across the barrier. The subscript $t$ denotes partial differentiation with respect to time. The equation for $\phi$ now becomes

$$
\frac{\hbar C}{2 e} \phi_{t t}+\frac{\hbar}{2 e R} \phi_{t}+I_{c 0} \sin \phi=I_{0}+I_{1} \cos \omega t
$$

where the externally applied current, $I(t)$, on the righthand side is taken to be in the specified form. Using normalized units it is possible to write Eq. (1) on the form

$$
\phi_{t t}+\alpha \phi_{t}+\sin \phi=i_{0}+i_{1} \cos \Omega t .
$$

The frequency unit is here the maximum plasma angular frequency $\omega_{p 0}=\left(2 e I_{c 0} / \hbar C\right)^{1 / 2}$ and the time is normalized to $\omega_{p 0}^{-1}$. Currents are in units of $I_{c 0}$ and the loss parameter $\alpha$ is equal to $\left(\omega_{p 0} R C\right)^{-1}$. The structure of Eq. (2) is similar to the equation for a damped pendulum in a gravitational field driven by an externally applied torque, $i(t)$. In the zero-voltage state we can linearize Eq. (2) by assuming $\phi(t) \approx \phi_{0}+\epsilon \phi_{1}(t)$, which is valid for a smallamplitude oscillating driving force, i.e., $i_{1} \ll 1$. Formally Eq. (2) now has the same form as the equation of motion for a driven damped linear resonance circuit:

$$
\phi_{1 t t}+\alpha \phi_{1 t}+\cos \phi_{0} \cdot \phi_{1}=i_{1} \cos \Omega t
$$

with the resonance frequency given by

$$
\Omega_{p}=\sqrt{\cos \phi_{0}}=\sqrt[4]{1-i_{0}^{2}}
$$

for $\alpha \ll 1$ (experimentally, $\alpha$ is typically of the order of $10^{-2}$ ). We will identify $\Omega_{p}$ with the (normalized) frequency of plasma oscillations in the linear, small amplitude limit.

If a static magnetic field $\mathbf{H}$ is present in the barrier, $\phi$ will vary spatially in accordance with the equation ${ }^{6}$

$$
\nabla \phi=\frac{2 \pi \mu_{0} d}{\Phi_{0}} \mathbf{H} \times \hat{\mathbf{n}} .
$$

Here the magnetic thickness of the barrier, $d=\lambda_{1}+\lambda_{2}+t_{\text {ox }}$, where the $\lambda$ 's are the London penetration depths for the two superconductors and $t_{\mathrm{ox}}$ is the thickness of the oxide barrier. The magnetic flux quan- tum is denoted by $\Phi_{0}=h / 2 e$. In general, the magnetic field can either be externally applied or generated by the Josephson current itself. We assume that the magnetic field $H_{y}$ is in the $y$ direction, so that $\phi$ depends only on the $x$ coordinate where $x-y$ defines the barrier plane, see Fig. 1. Following the analysis by Dahm et al., $\phi$ can be written as a sum of space-dependent and time-dependent parts $\phi(x, t) \approx \phi_{0}(x)+\epsilon \phi_{1}(t)$, where $\epsilon \phi_{1}$ is the small perturbation mentioned earlier.

Since the spatial distribution of the Josephson phase is neglected in the equation of motion for short junctions, the problem of calculating the plasma resonance frequency reduces to a matter of proper spatial averaging. This is equivalent to calculating the center-of-mass motion for a rigid body in classical mechanics. To account for the effect of the magnetic field the sine term in Eq. (1) is replaced by $\left\langle J_{c 0}(x, y) \sin \left[\phi_{0}(x)+\epsilon \phi_{1}(t)\right]\right\rangle$, where the angular brackets denote the spatial average over the junction area. The Josephson current is

$$
I_{0}=\left\langle J_{c 0}(x, y) \sin \phi_{0}(x)\right\rangle,
$$

where $J_{c 0}$ is the critical current density. Corresponding$1 \mathrm{y}$, the expression for the plasma frequency becomes

$$
\omega_{p}^{2}=\left\langle\omega_{p 0}^{2} \cos \phi_{0}(x)\right\rangle .
$$

If the magnetic field $H_{y}$ in the barrier is uniform Eq. (4) reduces to

$$
\phi_{0}(x)=\phi_{0}+\frac{2 \pi \mu_{0} d H_{y}}{\Phi_{0}} x,
$$

where $\phi_{0}$ is a constant of integration to be determined by the dc bias.

Without a great loss of generality we can take $J_{c 0}$ to be symmetric with respect to the $x$ direction. Carrying out the integration along the $y$ direction we can evaluate Eqs. (5) and (6) with the use of Eq. (7) and the notation $J_{S}(x)=J_{S}(-x)=\int J_{c 0}(x, y) d y$ :

$$
\begin{aligned}
I_{0}= & \sin \phi_{0} \int J_{S}(x) \cos \left[\frac{2 \pi \mu_{0} d H_{y} x}{\Phi_{0}}\right] d x \\
& +\cos \phi_{0} \int J_{S}(x) \sin \left[\frac{2 \pi \mu_{0} d H_{y} x}{\Phi_{0}}\right] d x \\
\omega_{p}^{2}= & \frac{2 e}{\hbar C}\left[\cos \phi_{0} \int J_{S}(x) \cos \left[\frac{2 \pi \mu_{0} d H_{y} x}{\Phi_{0}}\right] d x\right. \\
& \left.-\sin \phi_{0} \int J_{S}(x) \sin \left[\frac{2 \pi \mu_{0} d H_{y} x}{\Phi_{0}}\right] d x\right] .
\end{aligned}
$$

The last two terms in Eqs. (8) and (9) vanish by symmetric and the plasma frequency can be written as

$$
\omega_{p}^{2}=\frac{2 e f\left(H_{y}\right)}{\hbar C}\left[1-\left[\frac{I_{0}}{f\left(H_{y}\right)}\right]^{2}\right]^{1 / 2},
$$

where $f\left(H_{y}\right)=\int J_{S}(x) \cos \left(2 \pi \mu_{0} d H_{y} x / \Phi_{0}\right) d x$.

The antisymmetic last term in Eq. (8) describes a circulating current: 


$$
I_{\mathrm{cir}}=\frac{\cos \phi_{0}}{2} \int J_{S}(x)\left|\sin \left(\frac{2 \pi \mu_{0} d H_{y} x}{\Phi_{0}}\right)\right| d x .
$$

Pedersen, Finnegan, and Langenberg ${ }^{7}$ have tried to include the spatial dimension of the junction by keeping the equation of motion for the short junction and reduce the magnetic flux $\Phi_{y}$ by an amount $-L_{p} I_{\text {cir }}$, so $\Phi_{y}=\Phi_{\mathrm{ex}}-L_{p} I_{\text {cir }}$. Here, the geometrical inductance $L_{p} \approx \mu_{0} L d / W$ arises from the magnetic energy stored in the junction. In this model the additional flux $-L_{p} I_{\text {cir }}$, which depends on the bias current through $\cos \phi_{0}$, can affect the plasma frequency [see Eq. (10)], but not the measured critical current $I_{c}\left(H_{\mathrm{ex}}\right)$, since $I_{\mathrm{cir}}=0$ for $I_{0}=I_{c}\left(H_{\mathrm{ex}}\right)\left(\phi_{0}= \pm \pi / 2\right)$. In the short junction model self-fields are neglected and the function $f$ is replaced by $I_{c}\left(H_{\mathrm{ex}}\right)$, such that $H_{y}=H_{\mathrm{ex}}$.

For a homogeneous current density we can evaluate Eqs. (8), (9), and (11). A plot of $\Omega_{p}^{4}$ versus $i_{0}^{2}$ gives straight lines in accordance with Eq. (10) for $L_{p}=0$. In Fig. 2 we show a plot of this type for different values of $\Phi_{\mathrm{ex}}=\Phi_{y}+l_{p} i_{\text {cir }}$, where flux now is normalized to $\Phi_{0}$ and the inductance is in units of $\Phi_{0} / I_{c 0}$. The values of $l_{p}$ used are relevant for three of the measured samples.

\section{B. Perturbation analysis of long junctions}

For long junctions the spatial dimension of the tunneling structure must be taken into account. This is done by representing the junction as a lumped transmission-line equivalent of infinitesimally small RCSJ circuits, see Fig. 1. In the transmission-line model the individual RCSJ circuits are connected together by the inductance per unit length $L_{p} / L=\mu_{0} d / W$, see, for example, Ref. 8. The result is the perturbed sine-Gordon equation, where the additional term $\phi_{x x}$ originates from the inductance:

$$
\phi_{t t}-\phi_{x x}+\alpha \phi_{t}+\sin \phi=i_{0}+\epsilon i_{1} e^{i \Omega t} \text {. }
$$

Here the space coordinate $x$ is normalized to $\lambda_{J}=\left(\hbar / 2 e \mu_{0} d J_{c 0}\right)^{1 / 2}$. We have taken the applied current to be uniformly distributed along the length of the junction (overlap geometry). For simplicity we have neglected the surface losses in the superconducting films (the $\beta \phi_{x x t}$ term). The external magnetic field is accounted for through the boundary conditions: ${ }^{8} \quad \phi_{x}( \pm l / 2, t)=\eta$. Here, the (dimensionless) quantity $\eta$ is the external field in units of $J_{c 0} \lambda_{J}$ and $l=L / \lambda_{J}$.

In Sec. II A we treated the dynamics of plasma oscillations as a center-of-mass problem for a rigid chain of pendula. Since the mechanical analog to a long Josephson junction is a flappy chain of pendula, the approach is somewhat different. The purpose is to find the amplitude of the pendulum oscillations along the $x$ coordinate as a function of the external field.

The following perturbation analysis is valid for small values of the magnetic field $\eta$ and an oscillatory driving force $i_{1} \exp (i \Omega t)$. The bias current $i_{0}$ is set to zero, which is suitable for calculating the maximum plasma frequency in Sec. V. The small sinusoidal drive $\epsilon i_{1} \exp (i \Omega t)$ will cause a small perturbation in the Josephson phase, which we assume to be of the form $\phi(x, t) \approx \phi_{0}(x)+\epsilon \phi_{1}(x, t)$. In-

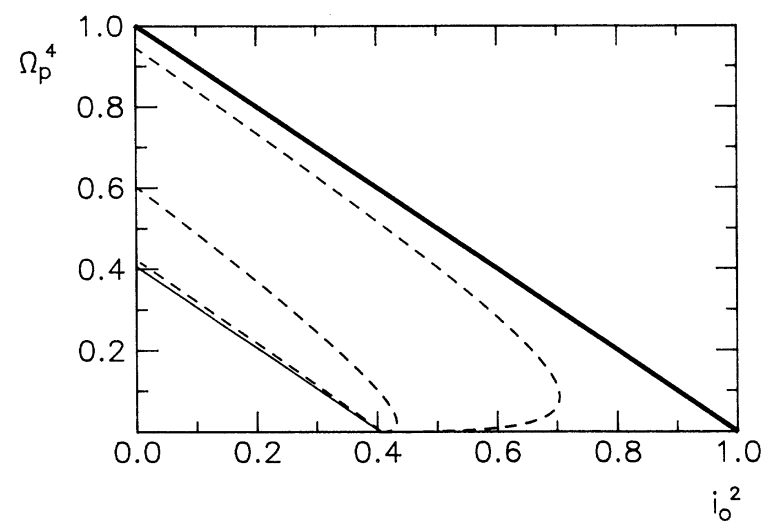

FIG. 2. Short junction model which accounts for the spatial dimension by introducing the (normalized) transmission line inductance $l_{p}$. The (normalized) magnetic flux in the barrier then becomes $\Phi_{y}=\Phi_{\mathrm{ex}}-l_{p} i_{\text {cir }}$ where $i_{\text {cir }}$ is a circulating current given by Eq. (11). The three dashed curves are for $\Phi_{\mathrm{ex}}=0.5$ and, from the bottom and upward: $l_{p}=0.065,0.22$, and 1.82 . The inductance values are relevant for samples $A, B$, and $C$, respectively. The thin solid line is for $l_{p}=0$ and $\Phi_{\mathrm{ex}}=0.5$, while the thick solid line represents $\Phi_{\mathrm{ex}}=0$ and all three inductance values.

sertion of this assumption into Eq. (12) yields

$$
\begin{aligned}
& -\phi_{0 x x}+\sin \phi_{0}(x)=0, \quad \phi_{0 x}( \pm l / 2)=\eta, \\
& \phi_{1 t t}-\phi_{1 x x}+\alpha \phi_{1 t}+\cos \phi_{0}(x) \cdot \phi_{1}=i_{1} e^{i \Omega t}, \\
& \phi_{1 x}( \pm l / 2, t)=0 .
\end{aligned}
$$

To solve Eqs. (13) and (14) we now impose the condition that $\eta$ and therefore $\phi_{0}$ is of the order $\epsilon$. Equation (13) is then linearized and the solution is [the corresponding expression for a short junction is given by Eq. (7)]

$$
\phi_{0}(x)=\eta \frac{\sinh (x)}{\cosh (l / 2)} \text {. }
$$

The stationary solution to Eq. (14) has the form $\phi_{1}(x, t)=\alpha(x) \exp (i \Omega t)$, where $a(x)$ is the complex amplitude of the plasma oscillations along the barrier. The equation for $a(x)$ can now be obtained by using the expansion $\cos \phi_{0} \approx 1-\phi_{0}^{2} / 2$, Eq. (15) and the abovementioned expression for $\phi_{1}$ :

$$
\begin{gathered}
a_{x x}-\left[1-\Omega^{2}+i \alpha \Omega-\epsilon^{2} \frac{\phi_{0}^{2}(x)}{2}\right] a=-i_{1}, \\
a_{x}( \pm l / 2)=0 .
\end{gathered}
$$

This equation is solved to first order by introducing $a(x) \approx a_{0}+\epsilon^{2} a_{1}(x)$. The equation for $a_{1}(x)$ then becomes

$$
\begin{gathered}
a_{1 x x}-\left(1-\Omega^{2}+i \alpha \Omega\right) a_{1}=-\frac{\phi_{0}^{2}(x)}{2} a_{0}, \\
a_{1 x}( \pm l / 2)=0,
\end{gathered}
$$

where $a_{0}=i_{1} /\left(1-\Omega^{2}+i \alpha \Omega\right)$, which is the well-known expression for a driven damped linear resonator. The final solution to our problem is 
$a(x)=a_{0}\left[1+\frac{\eta^{2}}{2\left(4-k^{2}\right) \cosh ^{2}(l / 2)}\left[\frac{\sinh (l) \cosh (k x)}{k \sinh (k l / 2)}-\sinh ^{2}(x)-\frac{2}{k^{2}}\right]\right]$.

Here we have introduced the complex number $k^{2}=1-\Omega^{2}+i \alpha \Omega$ with $k$ given by

$$
\begin{aligned}
& \operatorname{Re}(k)=\left[\frac{\left|k^{2}\right|+1-\Omega^{2}}{2}\right]^{1 / 2}, \\
& \operatorname{Im}(k)=\left[\frac{\left|k^{2}\right|-1+\Omega^{2}}{2}\right]^{1 / 2} .
\end{aligned}
$$

Figure 3 shows a typical plot of $|a(x)|$ and $\phi_{0}(x)$ together with the result of a numerical simulation of the sineGordon equation

\section{EXPERIMENTAL TECHNIQUES}

The samples used were $70 \times 70$ or $800 \times 20 \mu \mathrm{m}^{2}$ $\mathrm{Nb}-\mathrm{Nb}_{x} \mathrm{O}_{y}-\mathrm{Pb}$ tunnel junctions incorporated into a $50-\Omega$ microstrip structure on a Corning 7059 glass substrate. The thin film pattern is shown in Fig. 4. The microwave coupling to the sample was established through coaxial launchers at the ends of the microstrip. The microwave current used to stimulate the plasma resonance was provided from a microwave generator (a frequency synthesizer) connected to one side of the microstrip. In order to suppress microwave resonances, the other end was terminated by a $50-\Omega$ load at helium temperature. For

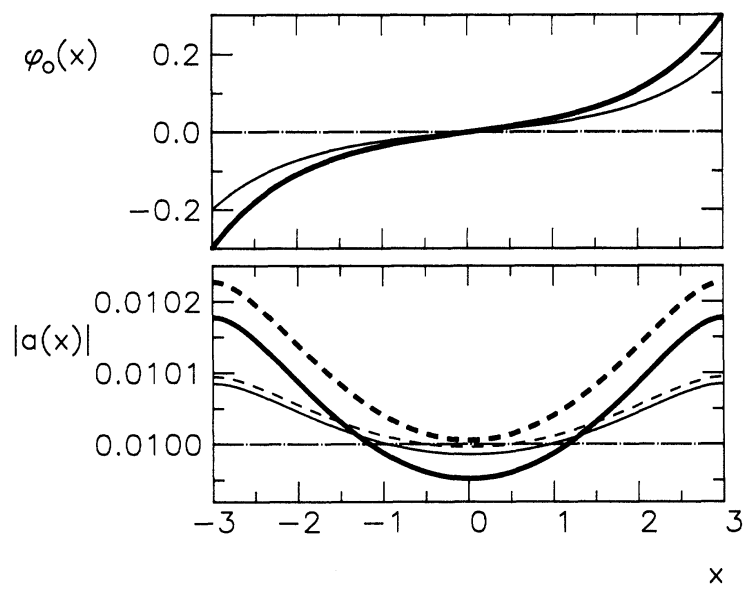

FIG. 3. The magnetic field perturbs the static distribution of the Josephson phase, $\phi_{0}(x)$, which then effects the dynamics of the plasma oscillations. The small-amplitude plasma oscillations, $a(x) \exp (i \Omega t)$, are to be superimposed on the stationary background shown in the top part of the figure. Dashed curves are legend for perturbation analysis and solid curve for full numerical simulation. Thick curves are for $\eta=0.3$ and thin cirves for $\eta=0.2$ (no visible difference between perturbation and simulation in the top part of the figure). The horizontal lines correspond to $\eta=0$. Other parameters are $\Omega=0.995, l=6, \alpha=0.1$, $i_{0}=0$, and $i_{1}=10^{-3}$. convenience the plasma resonance frequency was tuned by varying the bias current, $I_{0}$ [see Eq. (3)], while the frequency of the applied microwave current was fixed. Using this method the plasma resonance manifested itself as a dip in the reflected microwave signal as a function of $I_{0}$. A circular at room temperature separated the incoming signal from the reflected signal which was amplified and detected by two low-noise FET amplifiers followed by a digital spectrum analyzer (SA in Fig. 4).

The sample was placed in a vacuum can immersed in a pressure-regulated liquid helium bath. A pair of Helmholtz coils was used to create a static magnetic field. The current and voltage leads connected to the sample were carefully shielded and filtered against external noise sources. The ambient magnetic field was reduced by a double mu-metal shield and the entire experimental setup was placed in an rf-shielded room.

\section{EXPERIMENTAL RESULTS}

To ascertain that we only stimulated the plasma oscillations in the small amplitude limit, we decreased the microwave current, $I_{1}$, until the position of the resonance dip as a function of the bias current did not change with any further reduction of $I_{1}$. With the microwave coupling and detection system used the applied microwave power ranged from $10^{-15} \mathrm{~W}$ to $10^{-11} \mathrm{~W}$, corresponding to a normalized microwave current ranging from $10^{-7}$ to $10^{-5}$, see Ref. 5 .

From Eq. (3) it is clear that a plot of $f_{p}^{4}=\left(\omega_{p} / 2 \pi\right)^{4}$ versus $I_{0}^{2}$ should yield a straight line which intersects the
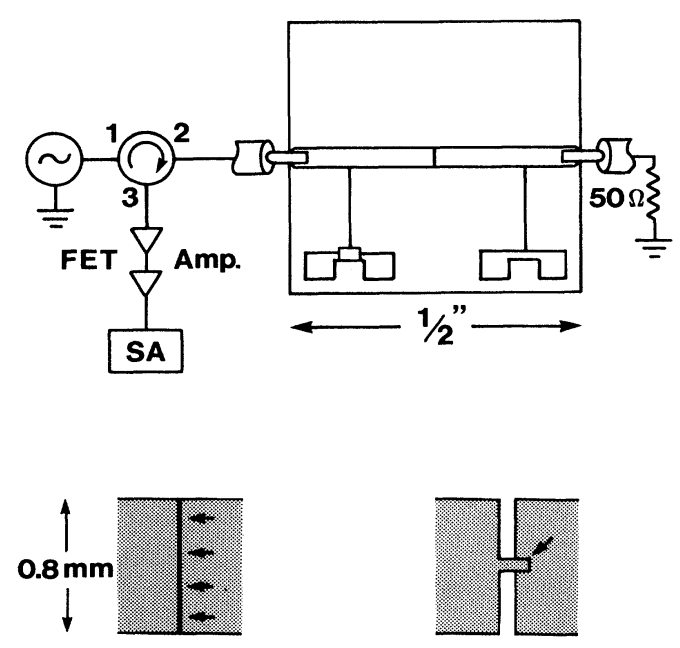

FIG. 4. Upper part: schematic of the experimental microwave system (SA stands for spectrum analyzer), including the thin film microstrip design. The enlarged bottom part shows the junction geometries (the overlap of the two superconducting films is indicated by arrows). 
axes in $f_{p 0}^{4}$ and $I_{c 0}^{2}$. Figure 5 shows a plot of this kind in the case of no applied magnetic field for a short junction. Measurements of the plasma resonance offer a precise way to determine the critical current and the capacitance. The values of $I_{c 0}$ found in this way are always a few percent larger than the value obtained by a slow sweep of the $I-V$ characteristics, where premature switching from the zero-voltage state, induced by thermal noise, gives a lower value on $I_{c 0}$.

In order to examine the effect of a static magnetic field on the dynamics of the junction we compared measurements for two ways of reducing $I_{c 0}$ - either by applying an external magnetic field or by raising the temperature $T$. For every value of $I_{c}\left(H_{e x}\right)$ or $I_{c}(T)$ a plot like Fig. 5 was made in order to find $f_{p 0}$ and $I_{c}$ (at least four wellspaced frequencies were used for the least-squares fit). The results are presented as a plot of $f_{p 0}^{2}$ versus $I_{c}$, where the theory for short junctions predicts a straight line through origo. The top graph in Fig. 7 shows the results for a short junction.

For longer junctions the behavior deviates from that of the short junction. In Fig. 6 we notice two important characteristics. First, the measured points to a very good approximation still lie on straight lines which intersect the $x$ axis in $I_{c}^{2}$. Second, and most important, the slope of the these lines changes with the applied field but not with the temperature. Since the $f_{p}^{4}$ versus $I_{0}^{2}$ data points fall on straight lines in Fig. 6 we feel justified in making a least-squares fit and extract informations about $f_{p 0}$ and $I_{c}$. The results are shown in the middle and bottom parts of Fig. 7. A plot of $f_{p 0}^{2}$, versus $I_{c}(T)$ should still yield a straight line through origo for long junctions with a $u n i$ form current distribution. The $I_{c}(T)$ series of measurements in Fig. 7 supports our assumption of a uniform current distribution.

There can be up to a $22 \%$ difference in plasma frequen-

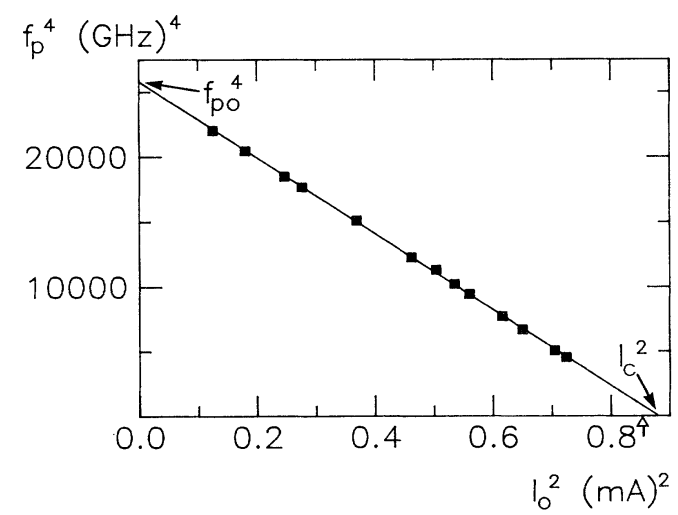

FIG. 5. Measured tuning of the plasma resonance frequency by the bias current in a short junction in the absence of a magnetic field, here plotted as $f_{p}^{4}$ vs $I_{0}^{2}$, see Eq. (3). The straight line is a least-squares fit. The intersections with the axes give $I_{c 0}(4.2$ $\mathrm{K})=938.9 \mu \mathrm{A}, f_{p 0}=12.67 \mathrm{GHz}$, and therefore $C=450 \mathrm{pF}$. The open arrow indicates the critical current obtained by a slow sweep of the bias current.

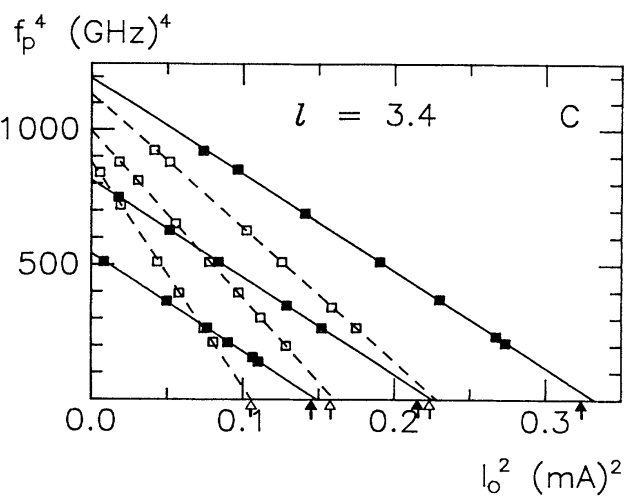

FIG. 6. Experimental data for the tuning of the plasma resonance frequency by the bias current in a long junction (sample $C)$. For comparison the critical current was reduced in two ways - either by applying a magnetic field $(\square)$ or by raising the temperature ( $\boldsymbol{\square})$. All lines are least-squares fits. The open and closed arrows indicate the critical current obtained by a slow sweep of the bias current for the $I_{c}\left(H_{\mathrm{ex}}\right)$ and $I_{c}(T)$ series, respectively.

cy between the cases where the critical current is tuned either by the magnetic field or by the temperature (bottom part of Fig. 7). In this example the normalized length was 3.4 and the critical current was decreased to $57 \%$ of $I_{c 0}$ at $4.2 \mathrm{~K}$. The corresponding reduction of $I_{c 0}$ for a junction of normalized length 1.1 was $7.5 \%$.

\section{NUMERICAL SIMULATIONS AND PERTURBATION RESULTS}

Numerical simulations of Eq. (12) with the boundary conditions $\phi_{x}( \pm l / 2, t)=\eta$ were obtained using an explicit difference scheme. A time rigid of 0.005 and a space grid of 0.02 were typically used in the integration. The loss parameter $\alpha$ was set to 0.1 , while the amplitude of the sinusoidal drive $i_{1}$ was $10^{-3}$ to ensure the linear, small signal limit of the plasma oscillations.

To compare the numerical simulations with the experiments we have to establish a relation between the calculations and the reflected signal measured by the detection system. A reasonable representation of the reflected sig-

TABLE I. Sample parameters at $4.2 \mathrm{~K}$ for three $\mathrm{Nb}-\mathrm{Nb}_{x} \mathrm{O}_{y}$ $\mathrm{Pb}$ junctions used in this study. $R_{N}$ is the normal state resistance.

\begin{tabular}{cccc}
\hline \hline Sample & $A$ & $B$ & $C$ \\
\hline$L \times W\left(\mu \mathrm{m}^{2}\right)$ & $71 \times 68$ & $71 \times 71$ & $776 \times 18$ \\
$I_{c 0}(\mu \mathrm{A})$ & 116.2 & 2427 & 576.5 \\
$f_{p 0}(\mathrm{GHz})$ & 4.49 & 18.38 & 5.88 \\
$C(\mathrm{pF})$ & 443 & 553 & 1283 \\
$R_{N}(\Omega)$ & 48.7 & 0.51 & 1.98 \\
$\lambda_{J}(\mu \mathrm{m})$ & 220 & 62 & 230 \\
$l$ & 0.3 & 1.1 & 3.4 \\
\hline \hline
\end{tabular}



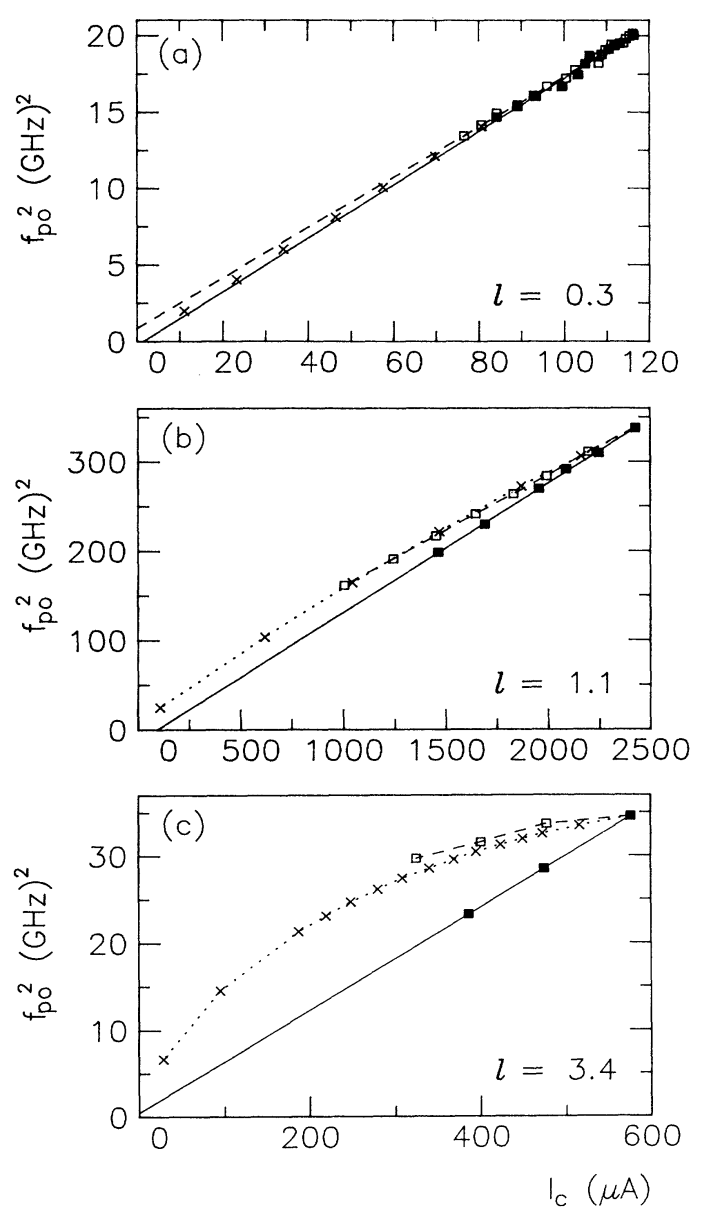

FIG. 7. Experimental results plotted as the squared maximum plasma frequency vs the critical current for junctions of various normalized lengths, $l$ [samples $A, B$, and $C$ in (a), (b), and (c), respectively]. The intersections with the axes in a $f_{p}^{4}$ vs $I_{0}^{2}$ plot were used to determine $f_{p 0}$ and $I_{c}$ (see Fig. 5). The critical current was reduced by either applying a magnetic field $(\square)$ or by raising the temperature ( $\boldsymbol{\square})$. All solid lines and the dashed line in the top part of the figure are least-squares fits. The crosses $(X)$ show the results of a numerical simulation of the perturbed sine-Gordon equation with the relevant normalized lengths.

nal would be the spatial average of the voltage, $\left\langle\phi_{t}\right\rangle$, along the length of the barrier. Throughout the paper the plasma resonance is defined as a resonance in $\phi$ and not in $\phi_{t}$. The difference in the resonance frequency introduced by finding the resonance point of $\phi_{t}$ instead of $\phi$ is around $\alpha^{2} / 4$ for small $\alpha$ values. For consistency, we therefore keep the definition of the resonance and calculate the spatial average of the oscillatory part, $\phi_{1}$, of the Josephson phase:

$$
\left\langle\phi_{1}\right\rangle=A(\Omega, \eta) \cos (\Omega t+\Gamma) \text {. }
$$

The resonance condition for $\left\langle\phi_{1}\right\rangle$ is obtained when the parameter $A$ in Eq. (18) is maximum. The simulations were carried out as in the experiment, i.e., the bias current was increased while $\Omega$ and $\eta$ were kept fixed. The results confirmed the linear dependence shown in Figs. 5 and 6 . The critical current and the maximum plasma frequency can be obtained as in the experiment by extrapolating a least-squares fit. A more precise and suitable way is to find the point of resonance for $\left\langle\phi_{1}\right\rangle$ by maximizing $A$ with respect to $\Omega$ for a given value of the magnetic field $\eta$. Doing this with a standard numerical procedure for $i_{0}=0$ we found the maximum plasma frequency as a function of $\eta$. The critical current was simply calculated by increasing the bias current until the phase started to rotate, which corresponds to the voltage state in the experiment. The value of the critical current determined in this way was equal to the extrapolated current value within the numeric resolution. The simulations were performed with the relevant normalized lengths and the results are presented in Fig. 7.

To use the perturbation result derived in Sec. II B we have evaluated $A^{2}=\langle\operatorname{Re}(a)\rangle^{2}+\langle\operatorname{Im}(a)\rangle^{2}$ numerically and maximized $A$ (also numerically) with respect to $\Omega$. This gives $\Omega_{p 0}$ and we only need an expression for the normalized critical current $i_{c}(\eta)=I_{c}(\eta) / I_{c 0}$ to make a plot of $\Omega_{p 0}^{2}$ versus $i_{c}$. We will only compare the perturbation result with numerical simulations for the two limiting cases of very short and very long junctions [we note that implicit expressions for $i_{c}(\eta)$ for overlap junctions of arbitrary lengths have recently been derived, Ref. 9]. The two limiting expressions are for very short junctions

$$
i_{c}(\eta)=\frac{\sin (l \eta / 2)}{\ln / 2}, l \ll 1
$$

and for very long junctions, ${ }^{10}$

$$
(\eta / 2)^{2}=\sqrt{1-i_{c}^{2}}-i_{c} \cos ^{-1} i_{c}, \quad l \gg 1 .
$$

The critical current equals zero when $\eta$ is equal to $2 \pi / l$ or 2 for short or long junctions, respectively.

\section{DISCUSSION}

The theoretical prediction of the RCSJ model for a short junction reproduced the experimentally measured tuning of the plasma frequency by the bias current and thereby confirmed the sinusoidal phase variation of the Josephson current. Within the available range of magnetic field a junction of normalized length $l=0.3$ (sample $A$ ) was sufficiently short, so that the spatial variation of the Josephson phase could be neglected in the governing equation of motion.

Two models are proposed to account for the deviation from the short junction behavior reported in Figs. 6 and 7. The model proposed by Pedersen et al. uses the short junction model in combination with the total inductance $L_{p}$. A $\Omega_{p}^{4}$ versus $i_{0}^{2}$ plot using this model is shown in Fig. 2 with the relevant inductances for samples $A, B$, and $C$. In our experiment the critical current was determined by an extrapolation of the least-squares fit to the $f_{p}^{4}$ versus $I_{0}^{2}$ data. If we use a similar extrapolation of the data points calculated from the model, the resulting intersection, $i_{i}$, is up to $45 \%$ larger than the critical current (e.g., for sample $C$ with $l_{p}=1.82$ and $\Phi_{\mathrm{ex}} / \Phi_{0}=0.5, i_{c} \approx 0.64$ 
and $\left.i_{i} \approx 0.93\right)$. Such a discrepancy was not seen in the experimental data, where the extrapolation of the $f_{p}^{4}$ versus $I_{0}^{2}$ data always gave a value of $I_{i}$ which was only a few percent larger than the $I_{c}$ measured by a slow sweep of the $I-V$ characteristic (e.g., $I_{i}-I_{c}=1 \%$ of $I_{c}$ for sample $C)$. As seen from Fig. 2 this model also predicts the existence of a finite lower limit on the accessible plasma frequency, since the experiments are restricted to the zerovoltage state $\left(I_{0}<I_{c}\right)$. Experimentally, the lower limit predicted by this model was not seen.

In contradistinction, the model proposed in this paper includes the inductance $L_{p}$ in the governing equation of motion. The numerical simulations reproduced quite well all the features seen in Fig. 6. For all three samples the agreement between theory and experiment in the $f_{p 0}^{2}$ versus $I_{c}$ plot in Fig. 7 is good.

As seen in Fig. 3 the result of the perturbation analysis gives a reasonable approximation to the spatial variation of the amplitude of the plasma oscillations. Minor devia-
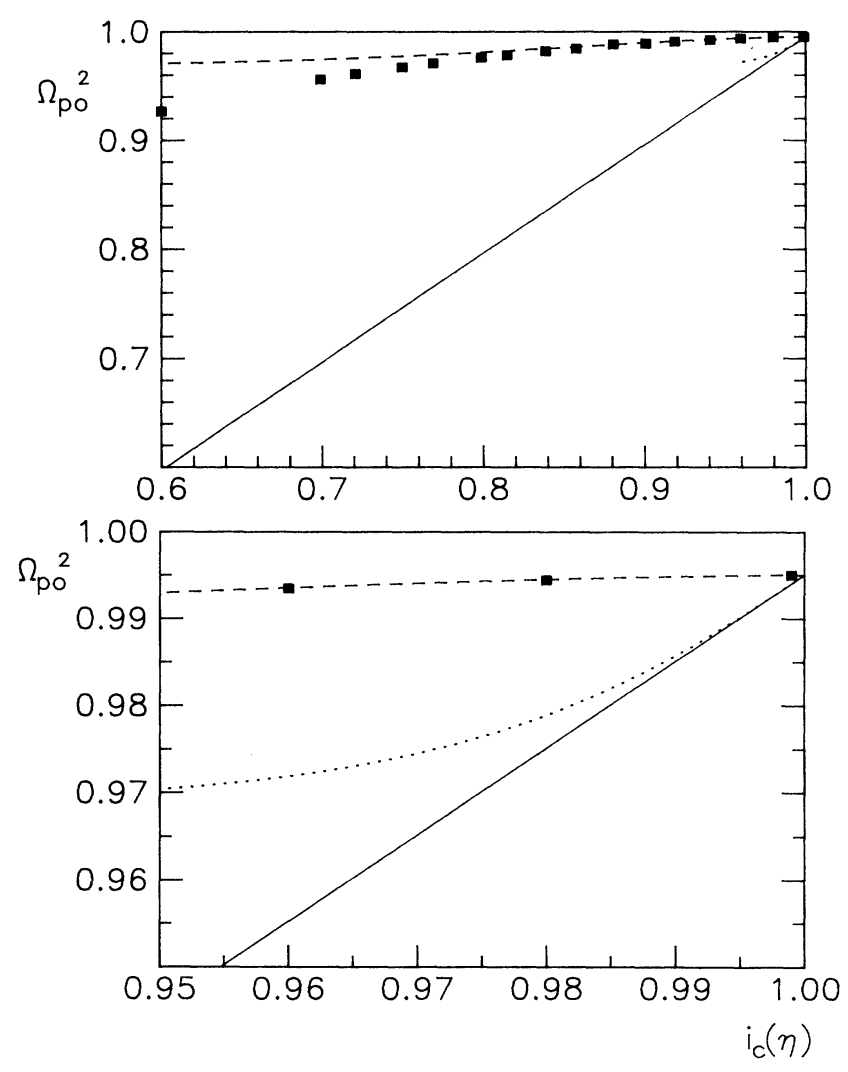

FIG. 8. A comparison between numerical simulations on the sine-Gordon system and the perturbation result for the two limiting cases of very short and very long junctions. The maximum plasma frequency is obtained by maximizing the parameter $A$ in Eq. (18), while the critical current is taken from Eqs. (19) and (20). The straight line represents the theoretical result for a short junction, while the dotted and dashed curves show the perturbation result for the two limiting cases $l=0.1$ and $l=10$, respectively. Solid squares are numerical simulations with $l=10, \alpha=0.1, i_{0}=0$, and $i_{1}=10^{-3}$. tions from the results of a full numerical simulation show up as offsets in the amplitude $a(x)$. The region of validity of the perturbation analysis is found from $\Omega_{p 0}^{2}$ versus $i_{c}(\eta)$ plots. As a criterion for the validity of the perturbation analysis we have calculated the value of the critical current where there is a $1 \%$ difference in frequency between the results of the simulation and the perturbation calculations. As seen in Fig. 8, we find that this difference exceeds $1 \%$ for $i_{c}<0.955$ for the very short junction and the very long one has $i_{c}<0.69$. The perturbation analysis is clearly more powerful for longer junctions.

\section{CONCLUSIONS}

We have experimentally demonstrated that the functional dependence of plasma oscillations in short tunnel junctions $\left(L / \lambda_{J}=0.3\right)$ is very well accounted for by the resistor-capacitor shunted junction model. This is the case when the critical current is decreased either by raising the temperature or by applying a magnetic field. If the junction is approximately one Josephson penetration depth long, deviations from the theory for short junctions start to show up. The deviations become more pronounced for still longer junctions. We emphasize that the above-mentioned deviation only appears in the presence of a magnetic field. More specifically, when an external magnetic field is applied, the square of the plasma frequency does not decrease as fast as the critical current. This again means that the plasma frequency is larger in a long junction exposed to a magnetic field than for the same junction with the critical current decreased by raising the temperature. An effect like this should show up as corrections in expressions involving the plasma frequency, for example, in the lifetime of the zero-voltage state.

Numerical simulations were performed on the governing sine-Gordon equation. They reproduced the experimental data quite well. This allows us to give the following qualitative interpretation of our results: the critical current is the bias current value, where the Josephson phase tilts over and starts to rotate. In other words, the critical current is a static property of the junction, whereas the plasma oscillation is obviously a dynamical property of the junction. A short junction can be treated as a rigid body, and it is not possible to distinguish between the two properties. For long junctions the static and the dynamic behavior in the above-mentioned sense become uncoupled. The critical current is determined by the amount of magnetic field at the boundaries. Since the field only penetrates a distance of the order of one Josephson penetration depth into the junction, the interior of the junction is not affected by the field. This leaves the plasma oscillations in the main part of the junction unperturbed by the magnetic field. Thus, as observed with increasing junction length the detuning of the plasma frequency with applied field becomes smaller.

Finally, in modeling the system we stress that it is important to include fully the spatial dimension of the Josephson phase in the governing equation of motion in 
order to account properly for the plasma oscillation dynamics in spatially extended Josephson tunnel junctions.

\section{ACKNOWLEDGMENTS}

We thank G. Friis Eriksen for valuable technical assistance and I. Rasmussen and S. Hjort for sample fabrica- tion. N. Grønbech-Jensen is also acknowledged for helpful advice on the simulations. One of us (T.H.) would like to thank the Carlsberg Foundation for financial support during the initial stages of this work. This work was partly supported by the Danish Natural Science Research Council.
${ }^{1}$ A. J. Dahm, A. Denenstein, T. F. Finnegan, D. N. Langenberg, and D. J. Scalapino, Phys. Rev. Lett. 20, 859 (1968).

${ }^{2}$ R. L. Kautz and R. Monaco, IEEE Trans. Mag. MAG-25, 1399 (1989).

${ }^{3}$ M. H. Devoret, J. M. Martinis, and J. Clarke, Phys. Rev. Lett. 53, 1260 (1984).

${ }^{4}$ A. J. Dahm and D. N. Langenberg, J. Low Temp. Phys. 19, 145 (1975); O. P. Balkashin and I. K. Yanson, Fiz. Nizk. Temp. 2, 289 (1976) [Sov. J. Low Temp. Phys. 2, 143 (1976)].

${ }^{5}$ T. Holst and J. Bindslev Hansen, Physica B 166, 1649 (1990).

${ }^{6}$ B. D. Josephson, Phys. Lett. 1, 251 (1962); B. D. Josephson, Adv. Phys. 14, 419 (1965).
${ }^{7}$ N. F. Pedersen, T. F. Finnegan, and D. N. Langenberg, Phys. Rev. B 6, 4151 (1972).

${ }^{8}$ P. S. Lomdahl, O. H. Soerensen, and P. L. Christiansen, Phys. Rev. B 25, 5737 (1982); N. F. Pedersen, in Solitons, edited by S. E. Trullinger, V. E. Zakharov, and V. L. Pokrovsky (North-Holland, Amsterdam, 1986), pp. 469-501.

${ }^{9}$ S. Pagano, B. Ruggiero, and E. Sarnelli, Phys. Rev. B 43, 5464 (1991).

${ }^{10}$ M. Yu. Kupriyanov, K. K. Likharev, and V. K. Semenov, Fiz. Nizk. Temp. 2, 1252 (1976) [Sov. J. Low Temp. Phys. 2, 610 (1976)]. 